\title{
Inverted exponentiated Weibull distribution with applications to lifetime data
}

\author{
Seunghyung Lee ${ }^{a}$, Yunhwan Noh ${ }^{a}$, Younshik Chung ${ }^{1, a}$ \\ ${ }^{a}$ Department of Statistics, Pusan National University, Korea
}

\begin{abstract}
In this paper, we introduce the inverted exponentiated Weibull (IEW) distribution which contains exponentiated inverted Weibull distribution, inverse Weibull (IW) distribution, and inverted exponentiated distribution as submodels. The proposed distribution is obtained by the inverse form of the exponentiated Weibull distribution. In particular, we explain that the proposed distribution can be interpreted by Marshall and Olkin's book (Lifetime Distributions: Structure of Non-parametric, Semiparametric, and Parametric Families, 2007, Springer) idea. We derive the cumulative distribution function and hazard function and calculate expression for its moment. The hazard function of the IEW distribution can be decreasing, increasing or bathtub-shaped. The maximum likelihood estimation (MLE) is obtained. Then we show the existence and uniqueness of MLE. We can also obtain the Bayesian estimation by using the Gibbs sampler with the Metropolis-Hastings algorithm. We also give applications with a simulated data set and two real data set to show the flexibility of the IEW distribution. Finally, conclusions are mentioned.

Keywords: Bayesian estimation, exponential inverted Weibull distribution, inverted exponentiated Weibull distribution, inverse Weibull distribution, Gibbs sampler, hazard function, maximum likelihood estimate, Metropolis-Hastings algorithm
\end{abstract}

\section{Introduction}

Mudholkar and Srivastava (1993) introduced a three-parameter exponentiated Weibull (EW) distribution as an extension of Weibull distribution. Singh et al. $(2002,2005)$ investigated Bayes estimators proposed for three-parameter EW distribution when the available sample is type-II censored. The Weibull and EW model are widely discussed for survival analysis because of its simplicity and flexible modelling.

In this paper, we propose a new distribution, referred to as the inverted exponentiated Weibull (IEW) distribution, which contains exponentiated inverted Weibull (EIW) distributions, inverse Weibull (IW) distribution, inverted exponentiated distribution, and generalized inverted exponential (GIE) distribution as special sub-models. If a random variable $X$ has an EW distribution, then $Y=1 / X$ is said to have IEW distribution. In particular, we explain that the proposed distribution can be interpreted by Marshall and Olkin's (2007) idea. Bayesian estimation of parameters of IW distribution was previously investigated by Singh et al. (2013). The hazard function of IEW distribution can be decreasing, increasing and bathtub-shaped. Several properties of IEW distribution such as quantiles and moment are studied. Maximum likelihood estimator (MLE) and Bayesian estimator are obtained. We also show the existence and uniqueness of MLE.

\footnotetext{
${ }^{1}$ Corresponding author: Department of Statistics, Pusan National University, 2, Busandaehak-ro 63beon-gil, Geumjeonggu, Busan 46241, Korea. E-mail: yschung@pusan.ac.kr
}

Published 31 May 2017 / journal homepage: http://csam.or.kr

(C) 2017 The Korean Statistical Society, and Korean International Statistical Society. All rights reserved. 
The paper is organized as follows. In Section 2, we propose the IEW distribution and demonstrate the graphs of IEW distribution. In Section 3, we introduce some properties of IEW distribution such as cumulative distribution function (cdf), survivor function, hazard functions, quantiles, and moments. In Section 4, we obtain the MLE using the Newton Raphson method and Bayesian estimators using the Markov chain Monte Carlo (MCMC) method. In particular, we show the existence and uniqueness of MLE. In Section 5, we compare the performance of MLE and Bayesian estimates based on simulation studies. In addition, real data applications are performed. Finally, conclusions are mentioned in Section 6.

\section{Inverted exponentiated Weibull (EW) distribution}

Suppose that $Y$ has the EW distribution, $\operatorname{EW}(\alpha, \beta, \lambda)$ with $\alpha>0, \beta>0$, and $\lambda>0$ proposed by Mudholkar and Srivastava (1993) and its probability density distribution (pdf) and cdf are given by

$$
t(y ; \alpha, \beta, \lambda)=\alpha \beta \lambda y^{a-1} e^{-\lambda y^{\alpha}}\left(1-e^{-\lambda y^{\alpha}}\right)^{\beta-1}
$$

and

$$
T(y ; \alpha, \beta, \lambda)=\left(1-e^{-\lambda y^{\alpha}}\right)^{\beta},
$$

respectively. If $Y$ has the $\mathrm{EW}(\alpha, \beta, \lambda)$ distribution, then $X=1 / Y$ has the pdf given by

$$
f_{X}(x)=\alpha \beta \lambda x^{-(\alpha+1)} e^{-\lambda x^{-\alpha}}\left(1-e^{-\lambda x^{-\alpha}}\right)^{\beta-1} .
$$

This distribution in (2.3) is called the IEW distribution, denoted by $\operatorname{IEW}(\alpha, \beta, \lambda)$. The inverted exponentiated Rayleigh distribution proposed by Rastogi and Tripathi (2014) is a special case of the IEW distribution for $\alpha=2$. The IEW distribution also contains as sub-models EIW distribution for $\beta=1$ proposed by Flaih et al. (2012), GIE distribution proposed by Krishna and Kumar (2013) for $\alpha=1$ and IW distribution for $\beta=1$ and $\lambda=1$.

Following Marshall and Olkin (2007), we can interpret the $\operatorname{IEW}(\alpha, \beta, \lambda)$ in $(2.3)$ as:

Let $G_{\mathrm{IW}}(x)$ be the IW distribution given by

$$
G_{\mathrm{IW}}(x \mid \lambda, \alpha)=\exp \left[-\lambda x^{-\alpha}\right], \quad x>0
$$

where $\lambda>0$ and $\alpha>0$. The corresponding pdf is

$$
g_{\mathrm{IW}}(x \mid \lambda, \alpha)=\lambda \alpha x^{-(\alpha+1)} \exp \left[-\lambda x^{-\alpha}\right] .
$$

Marshall and Oklin (2007) pointed out that that the same kind of parameter does not expand the family of the distribution function. Then, Elbatal et al. (2016) mentioned that the resilience parameter can be used for the IW model since IW is a proportional reversed hazard family. Therefore, we can define the generalized inverse Weibull (GIW) distribution by applying the power of $\beta>0$ to $G_{\mathrm{IW}}(x)$ in (2.4), say

$$
\begin{aligned}
G_{\mathrm{GIW}}(x) & =1-\left(1-G_{\mathrm{IW}}(x)\right)^{\beta} \\
& =1-\left(1-e^{-\lambda x^{-\alpha}}\right)^{\beta}, \quad x>0,
\end{aligned}
$$



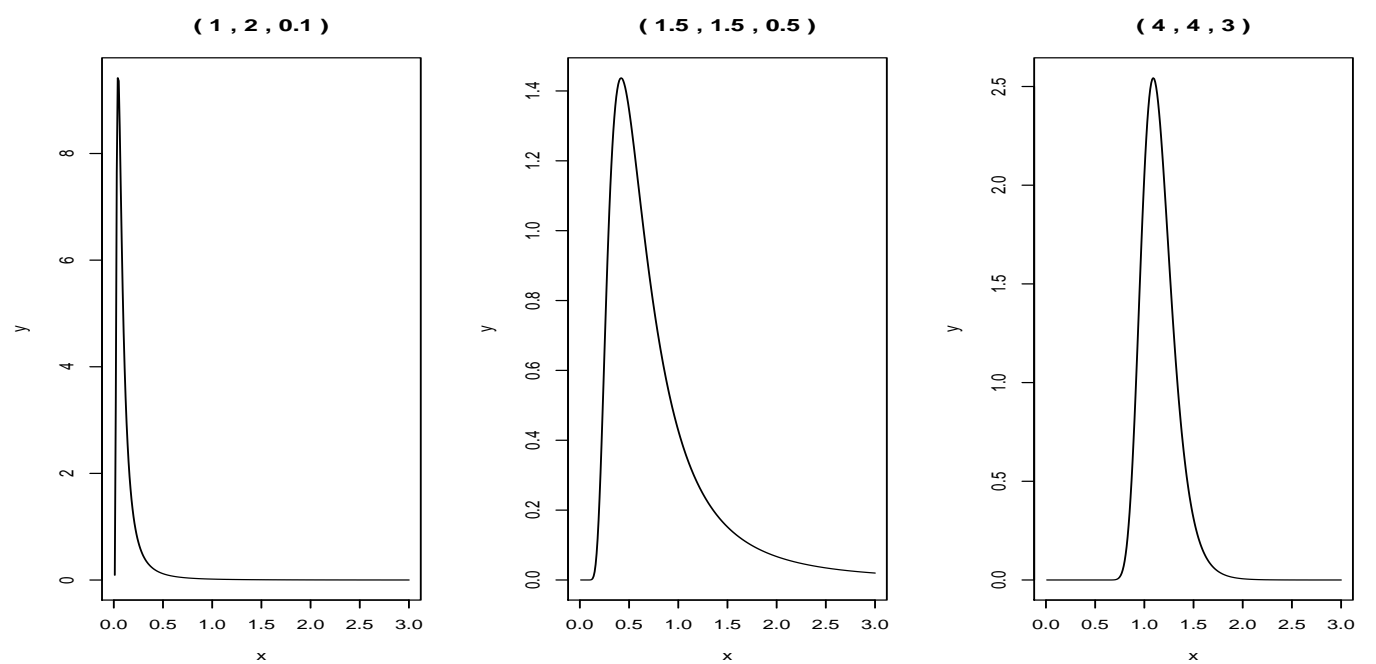

Figure 1: Probability density distribution of the inverse exponentiated Weibull distribution for selected values of the parameters.

which is the proportional hazard model, denoted by $\operatorname{GIW}(\alpha, \beta, \lambda)$. Hence the corresponding pdf of GIW distribution is given by

$$
\begin{aligned}
g_{\mathrm{GIW}}(x) & =\beta g_{\mathrm{IW}}(x)\left(1-G_{\mathrm{IW}}(x)\right)^{\beta-1} \\
& =\alpha \beta \lambda x^{-(\alpha+1)} e^{-\lambda x^{-\alpha}}\left[1-e^{-\lambda x^{-\alpha}}\right]^{\beta-1}, \quad x>0 .
\end{aligned}
$$

This $\operatorname{GIW}(\alpha, \beta, \lambda)$ in $(2.7)$ is the same as $\operatorname{IEW}(\alpha, \beta, \lambda)$ distribution in (2.3) that was originally proposed in this paper. Recently, de Gusmão et al. (2011) have tried to generalize the IW distribution by introducing the resilience parameter, but, as highlighted by Jones (2012), Gusmao et al. (2012) just proposed the distribution in (2.3) as the named Lehmann type II IW distribution without mentioning its properties and estimations.

Recall that the pdf and cumulative distribution of $\operatorname{EIW}(\alpha, \lambda)$ are given by

$$
g(y ; \alpha, \lambda)=\alpha \lambda y^{-(\alpha+1)} e^{-\lambda y^{-\alpha}}
$$

and

$$
G(y ; \alpha, \lambda)=e^{-\lambda y^{-\alpha}}
$$

respectively. Figure 1 shows the IEW density for various values of the parameter $(\alpha, \beta, \lambda)$.

If $|z|<1$ and $k>0$, we have the series representation

$$
(1-z)^{k-1}=\sum_{j=0}^{\infty} \frac{(-1)^{j} \Gamma(k)}{\Gamma(k-j) j !} z^{j},
$$

where $\Gamma(\cdot)$ is the gamma function. 
Expanding $\left(1-e^{-\lambda x^{-\alpha}}\right)^{\beta-1}$ as in (2.6), we can write (2.3) as

$$
\begin{aligned}
f(x ; \alpha, \beta, \lambda) & =\alpha \beta \lambda x^{-(\alpha+1)} e^{-\lambda x^{-\alpha}} \sum_{j=0}^{\infty} \frac{\Gamma(\beta)}{\Gamma(\beta-j) j !}(-1)^{j} e^{-\lambda j x^{-\alpha}} \\
& =\sum_{j=0}^{\infty}(-1)^{j} \beta \frac{\Gamma(\beta)}{\Gamma(\beta-j) j !} g(x ; \alpha, \lambda(j+1)),
\end{aligned}
$$

where $g(x ; \alpha, \lambda(j+1))$ is defined in (2.4). This means that the pdf of the IEW distribution can be expressed as an infinite mixture of EIW distribution with parameters $\alpha$ and $\lambda(j+1)$. Hence, some mathematical properties (cdf, moments, percentiles, moment generating function, and factorial moments) of the IEW distribution can be obtained using (2.7) from the corresponding properties of the EIW distribution.

\section{Properties of the inverse exponentiated Weibull (IEW) distribution}

\subsection{The distribution and hazard rate function}

Let $X$ be distributed to IEW distribution with parameters $(\alpha, \beta, \lambda)$ in $(2.3)$, that is,

$$
X \sim \operatorname{IEW}(\alpha, \beta, \lambda) .
$$

Its cdf is given by

$$
F(x)=1-\left(1-e^{-\lambda x^{-\alpha}}\right)^{\beta}, \quad x>0,
$$

which was proposed first by de Gusmão et al. (2012).

The survivor and hazard functions are

$$
S(x)=\left(1-e^{-\lambda x^{-\alpha}}\right)^{\beta}, \quad x>0
$$

and

$$
h(x)=\frac{\alpha \beta \lambda x^{-\alpha-1} e^{-\lambda x^{-\alpha}}\left(1-e^{-\lambda x^{-\alpha}}\right)^{\beta-1}}{\left(1-e^{-\lambda x^{-\alpha}}\right)^{\beta}},
$$

respectively. The limiting behavior of the hazard rate function given by (3.3) are: $\lim _{x \rightarrow \infty} h(x)=0$ and $\lim _{x \rightarrow 0} h(x)=\infty$. We illustrate some of the possible shapes of the hazard function for selected values of the $(\alpha, \beta, \lambda)$ in Figure 2. These plots show that the hazard rate function of the IEW distribution is nonmonotone and is very applicable.

\subsection{Quantiles and moments}

The quantile $u\left(x_{u}\right)$ of the IEW distribution follows from (2.8) as

$$
x_{u}=\left(\frac{1}{\lambda} \log \left(\frac{1}{1-(1-u)^{\beta}}\right)\right)^{-\frac{1}{\alpha}} .
$$

In particular, the median is simply calculated as $x_{0.5}=\left[(1 / \lambda) \log \left(1 /\left(1-0.5^{\beta}\right)\right)\right]^{-1 / \alpha}$. 

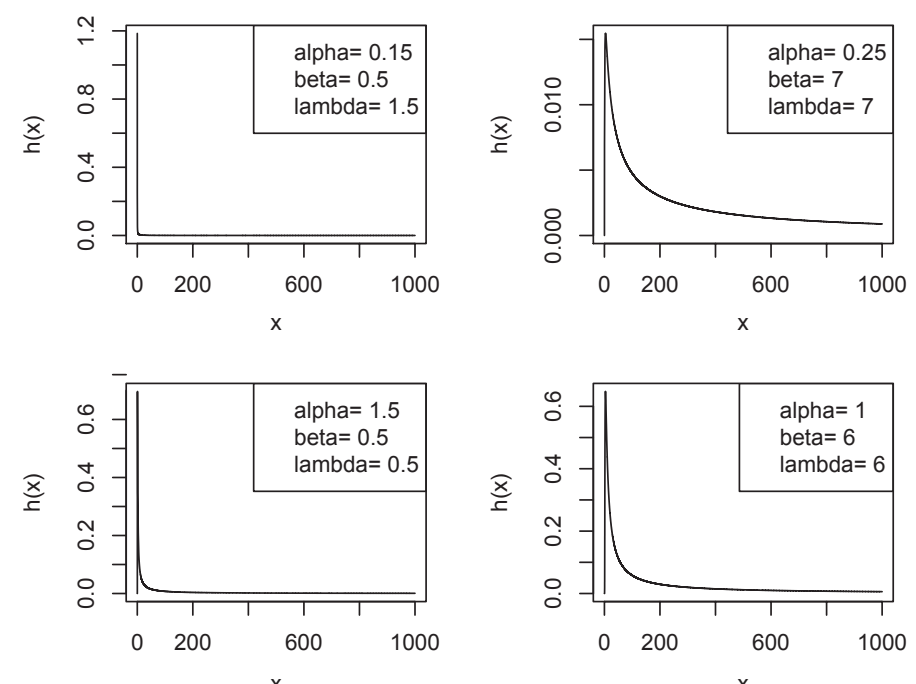

Figure 2: Hazard rate functions of the inverse exponentiated Weibull distribution for several values of the parameters.

Suppose that $Y \sim \operatorname{EIW}(\alpha, \lambda)$ in (2.4). Then $r^{\text {th }}$ moment of $Y$ is given by

$$
E\left[Y^{r}\right]=\lambda^{\frac{r}{\alpha}} \Gamma\left(1-\frac{r}{\alpha}\right), \quad \alpha>r
$$

Suppose that $X \sim \operatorname{EIW}(\alpha, \beta, \lambda)$. Then it follows from (2.11) that the $r^{\text {th }}$ moment of $X$ is given by

$$
\begin{aligned}
E\left[X^{r}\right] & =\int_{0}^{\infty} x^{r} \sum_{j=1}^{\infty}(-1)^{j} \beta \frac{\Gamma(\beta)}{\Gamma(\beta-j) j !} g(x ; \alpha, \lambda(j+1)) d x \\
& =\sum_{j=1}^{\infty}(-1)^{j} \beta \frac{\Gamma(\beta)}{\Gamma(\beta-j) j !}(\lambda(j+1))^{\frac{r}{\alpha}} \Gamma\left(1-\frac{r}{\alpha}\right), \quad \alpha>r .
\end{aligned}
$$

Using (3.5), the moment generating function of the IEW distribution is given by

$$
\begin{aligned}
M_{X}(t) & =E\left[\sum_{r=0}^{\infty} \frac{t^{r}}{r !} X^{r}\right] \\
& =\sum_{r=0}^{\infty} \sum_{j=0}^{\infty} \frac{t^{r}}{r !}(-1)^{j} \beta \frac{\Gamma(\beta)}{\Gamma(\beta-j) j !}(\lambda(j+1))^{\frac{r}{\alpha}} \Gamma\left(1-\frac{r}{\alpha}\right), \quad \alpha>r .
\end{aligned}
$$

For its other properties such as mean deviations, Rényi and Shannon entropies, Bonferroni and Lorenz curves, and order statistics, see Lee (2014). 


\section{Estimation}

\subsection{Maximum likelihood estimation (MLE)}

Let $\underline{x}=\left(x_{1}, \ldots, x_{n}\right)$ be a random sample from the IEW distribution in (2.3) with unknown parameter vector $\theta=(\alpha, \beta, \lambda)^{\prime}$. Then the $\log$ likelihood function $\ell=\ell(\theta ; x)$ for $\theta$ is

$$
\ell=\sum_{i=1}^{n}\left[-(\alpha+1) \log x_{i}-\lambda x_{i}^{-\alpha}+(\beta-1) \log \left(1-e^{-\lambda x_{i}^{-\alpha}}\right)\right]+n \log \alpha+n \log \beta+n \log \lambda
$$

The score function $U(\theta)=[(\partial \ell / \partial \alpha),(\partial \ell / \partial \beta),(\partial \ell / \partial \lambda)]^{T}$ based on a random sample of the IEW distribution in (2.3) has components as:

$$
\begin{aligned}
& \frac{\partial \ell}{\partial \alpha}=\frac{n}{\alpha}-\sum_{i=1}^{n} \log x_{i}+\sum_{i=1}^{n} \lambda x_{i}^{-\alpha} \log x_{i}-(\beta-1) \sum_{i=1}^{n} \frac{\lambda x_{i}^{-\alpha} \log x_{i} e^{-\lambda x_{i}^{-\alpha}}}{1-e^{-\lambda x_{i}^{-\alpha}}} \\
& \frac{\partial \ell}{\partial \beta}=\frac{n}{\beta}+\sum_{i=1}^{n} \log \left(1-e^{-\lambda x_{i}^{-\alpha}}\right)
\end{aligned}
$$

and

$$
\frac{\partial \ell}{\partial \lambda}=\frac{n}{\lambda}-\sum_{i=1}^{n} x_{i}^{-\alpha}+(\beta-1) \sum_{i=1}^{n} \frac{x_{i}^{-\alpha} \log x_{i} e^{-\lambda x_{i}^{-\alpha}}}{1-e^{-\lambda x_{i}^{-\alpha}}}
$$

The MLE $\hat{\theta}$ of $\theta$ is calculated numerically from the nonlinear equations $U(\theta)=0$. Here, we use the Newton Raphson method to find $\hat{\theta}$.

Next, we consider the asymptotic variances and covariances of MLEs. Then the asymptotic variances of MLEs are given by the inverse of the Fisher information matrix $I$. Unfortunately, the exact mathematical expressions for the Fisher information matrix are difficult to obtain. Then the observed Fisher information matrix $J(\theta)$ can be used instead of the Fisher information matrix. Therefore, we make an inference $\hat{\theta} \sim N\left(\theta, J^{-1}(\hat{\theta})\right)$ asymptotically where $J^{-1}(\hat{\theta})$ is the observed Hessian matrix evaluated at $\hat{\theta}$. So, the asymptotic normality of the MLE can compute the approximate $100(1-\alpha) \%$ confidence intervals for the parameters $\alpha, \beta$ and $\lambda$.

Finally, we consider the existence and uniqueness of the MLEs under some conditions and the proofs are given in Appendix.

Theorem 1. Let $g_{1}(\alpha ; \beta, \lambda)$ denote the function on the right hand side (RHS) of the equation (4.1) where $\beta$ and $\lambda$ are the true values of the parameters, then, the equation $g_{1}(\alpha ; \beta, \lambda)=0$ has at least one root for $\beta \neq 0$ and for $\beta=1$ the roots lie in the interval $\left[n /\left((1-\lambda) \sum_{i=1}^{n} \log x_{i}\right), n /\left(\sum_{i=1}^{n} \log x_{i}\right)\right]$, where $x_{i}>1$ and $\lambda<1$.

Theorem 2. Let $g_{2}(\beta ; \alpha, \lambda)$ denote the function on the RHS of the equation (4.2) where $\alpha$ and $\lambda$ are the true values of the parameters, then, the equation $g_{2}(\beta ; \alpha, \lambda)=0$ has at least one root and is unique.

Theorem 3. Let $g_{3}(\lambda ; \alpha, \beta)$ denote the function on the RHS of the equation (4.3) where $\alpha$ and $\beta$ are the true values of the parameters, then, the equation $g_{3}(\lambda ; \alpha, \beta)=0$ has at least one root. 


\subsection{Bayesian estimation}

Assume that $\alpha, \beta$, and $\lambda$ are independent. Each priors for $\alpha, \beta$, and $\lambda$ are assumed to be independent gamma distribution as:

$$
\pi_{1}(\alpha)=\frac{b_{1}^{a_{1}}}{\Gamma\left(a_{1}\right)} \alpha^{a_{1}-1} e^{-b_{1} \alpha}, \quad \pi_{2}(\beta)=\frac{b_{2}^{a_{2}}}{\Gamma\left(a_{2}\right)} \beta^{a_{2}-1} e^{-b_{2} \beta},
$$

and

$$
\pi_{3}(\lambda)=\frac{b_{3}^{a_{3}}}{\Gamma\left(a_{3}\right)} \lambda^{a_{3}-1} e^{-b_{3} \lambda}
$$

where $a_{i}$ and $b_{i}$ are assumed to be known for $i=1,2,3$.

The joint posterior density for $\alpha, \beta$, and $\lambda$ given the observed data $x_{1}, \ldots, x_{n}$ from IEW in (2.3) is obtained as:

$$
\pi\left(\alpha, \beta, \lambda \mid x_{1}, \ldots, x_{n}\right) \propto \prod_{i=1}^{n} \alpha \beta \lambda x_{i}^{-\alpha-1} e^{-\lambda x_{i}^{-\alpha}}\left(1-e^{-\lambda x_{i}^{-\alpha}}\right)^{\beta-1} \times \alpha^{a_{1}-1} e^{b_{1} \alpha} \beta^{a_{2}-1} e^{b_{2} \beta} \lambda^{a_{3}-1} e^{b_{3} \lambda}
$$

Therefore, for Gibbs sampling (Gelfand and Smith, 1990) the full conditional distributions (FCD) are obtained from (4.5) as:

$$
\begin{aligned}
& \pi\left(\alpha \mid \beta \lambda, x_{1}, \ldots, x_{n}\right) \propto \alpha^{a_{1}+n-1} e^{-\left(b_{1}+\sum_{i=1}^{n} \log x_{i}\right) \alpha} e^{-\lambda \sum_{i=1}^{n} x_{i}^{-\alpha}} \times \prod_{i=1}^{n}\left(1-e^{\left.-\lambda x_{i}^{-\alpha}\right)^{\beta-1}},\right. \\
& \pi\left(\beta \mid \alpha \lambda, x_{1}, \ldots, x_{n}\right) \propto \beta^{a_{2}+n-1} e^{-\left(b_{2}-\sum_{i=1}^{n} \log \left(1-e^{-\lambda x_{i}^{\alpha}}\right)\right) \beta}
\end{aligned}
$$

and

$$
\pi\left(\lambda \mid \alpha, \beta, x_{1}, \ldots, x_{n}\right) \propto \lambda^{a_{3}+n-1} e^{-\left(b_{3}+\sum_{i=1}^{n} x_{i}^{\alpha}\right) \lambda} \prod_{i=1}^{n}\left(1-e^{-\lambda x_{i}^{-\alpha}}\right)^{\beta-1} .
$$

However both FCD of $\alpha$ and $\lambda$ in (4.6) and (4.8) can not be reduced to well-known distributions, respectively and therefore it is impossible to sample directly by a standard methods. As suggested by Chib and Greenberg (1995), a hybrid MCMC algorithm is used by a combined Metropolis-Hastings sampling with Gibbs sampler using the suitable proposal distributions. To generate samples of $\alpha$ and $\lambda$ from (4.6) and (4.8), $G\left(n+a_{1},-\left(b_{1}+\sum_{i=1}^{n} \log x_{i}\right)\right)$ and $G\left(n+a_{3},-\left(b_{3}+\sum_{i=1}^{n} x_{i}^{\alpha}\right)\right)$ distributions are employed as their proposal distributions, respectively where $G(a, b)$ denotes the gamma distribution with the mean $a / b$. The hybrid MCMC algorithm is working as:

Step 1. Start with initial point $\left(\beta^{(0)}, \lambda^{(0)}\right)$.

Step 2. Set $j=1$.

Step 3. Using Metropolis-Hastings algorithm, generate $\alpha^{(j)}$ from $\pi\left(\alpha \mid \beta^{(j-1)}, \lambda^{(j-1)}, x_{1}, \ldots, x_{n}\right)$ in (4.6) with gamma proposal distribution, $G\left(n+a_{1},-\left(b_{1}+\sum_{i=1}^{n} \log x_{i}\right)\right)$.

Step 4. Generate $\beta^{(j)}$ from $G\left(n+a_{2}, b_{2}-\sum_{i=1}^{n} \log \left(1-e^{-\lambda^{(j-1)} x_{i}^{(j)}}\right)\right)$ in (4.7). 
Step 5. Using Metropolis-Hastings algorithm, generate $\lambda^{(j)}$ from $\pi\left(\alpha \mid \beta^{(j)}, \lambda^{(j)}, x_{1}, \ldots, x_{n}\right)$ in (4.8) with gamma proposal distribution, $G\left(n+a_{3},-\left(b_{3}+\sum_{i=1}^{n} x_{i}^{\alpha}\right)\right)$.

Step 6. Set $j=j+1$.

Step 7. Repeat Steps 3-7, $N$ times.

For example, the posterior mean of $\alpha$ is then approximated by

$$
\hat{\alpha}=\frac{1}{T-S} \sum_{i=S+1}^{T} \alpha^{(i)}
$$

where $S$ is the burn-in period. Here, we decide the convergence to have been reached after $S$ iteratins of and MCMC algorithm have been performed. Then the observations $\alpha^{(1)}, \alpha^{(2)}, \ldots, \alpha^{(S)}$ are discarded and $\alpha^{(i)}, S+1 \leq i \leq T$ are worked which are regarded as an independent sample from the stationary distribution of the Markov chain which is typically the posterior distribution.

\section{Applications}

\subsection{Simulation study}

In this study, we compare the performances of MLEs using the Newton Raphson method and Bayesian estimates using the MCMC method. To explain these, we conduct a simulation study using random sampling numbers generated from $\operatorname{IEW}(\alpha, \beta, \lambda)$ distribution with $\alpha=2.5, \beta=2.5$, and $\lambda=2.5$. We then use $n=30$ as sample size. Assume that the number of repetition is 10,000; subsequently, we calculate their means and standard errors of MLEs of each parameter. Therefore, we could calculate the corresponding root mean square errors (RMSE) of MLEs as:

$$
\operatorname{RMSE}=\sqrt{\frac{1}{10000} \sum_{i=1}^{10000}\left(\hat{\theta}^{(i)}-\theta\right)^{2}},
$$

where $\hat{\theta}^{(i)}$ denotes the MLE of the parameter $\theta$ at the $i^{t h}$ repetition. Next, for Bayesian estimations, each prior for $\alpha, \beta$, and $\lambda$ is assumed to be gamma distribution in (4.4) with parameters $a_{1}=a_{2}=a_{3}=$ 5 and $b_{1}=b_{2}=b_{3}=2$ since their mean values in simulation are assumed to be 2.5 . After checking the convergence, the number of iterations of Gibbs sampling and Metropolis-Hastings algorithm to be needed are 1,000, say $T-S$ in (4.9).

In Table 1, LCI and CP denote the length of $95 \%$ confidence intervals and its coverage probability of each parameters, respectively. LCI in Bayesian estimates means the length of highest posterior density (HPD) intervals. SE in parenthesis also stands for the standard error. For the simulated data from IEW model as above, All Bayesian estimates are better than the corresponding MLEs of each parameters in the senses of SE, RMSE, and CP. But, in the values of LCI, the lengths of confidence intervals of $\alpha$ and $\lambda$ was shorter than their corresponding HPD intervals.

Next, we compared IEW model with the competing models, such as EIW, EW, IW, transmuted Weibull (TW), and transmuted generalized exponential (TGE) models. TW distribution and TGE distribution are extended from the Weibull distribution and the generalized exponential distribution to analyze more complex data given by

$$
f_{\mathrm{TW}}(x \mid \alpha, \beta, \lambda)=\frac{\alpha}{\beta}\left(\frac{x}{\beta}\right)^{\alpha-1} e^{-\left(\frac{x}{\beta}\right)^{\alpha}}\left[1-\lambda+2 \lambda e^{-\left(\frac{x}{\beta}\right)^{\alpha}}\right],
$$


Table 1: MLEs and Bayesian estimates

\begin{tabular}{cccccc}
\hline \hline Parameter & Types & $\begin{array}{c}\text { Estimates } \\
(\mathrm{SE})\end{array}$ & RMSE & LCI & CP \\
\hline \multirow{2}{*}{$\alpha$} & MLEs & $\begin{array}{c}2.5861 \\
(0.3973)\end{array}$ & 0.1651 & 0.8645 & 0.7095 \\
\cline { 2 - 6 } & Bayesian estimates & $\begin{array}{c}2.5526 \\
(0.5540)\end{array}$ & 0.002 & 1.8028 & 0.9920 \\
\hline \multirow{2}{*}{$\beta$} & MLEs & $\begin{array}{c}2.6482 \\
(0.3844)\end{array}$ & 0.1696 & 3.1052 & 0.9738 \\
\cline { 2 - 6 } & Bayesian estimates & $\begin{array}{c}2.3613 \\
(0.2227)\end{array}$ & 0.005 & 2.6737 & 0.9920 \\
\hline \multirow{2}{*}{$\lambda$} & MLEs & $\begin{array}{c}2.5886 \\
(0.4323)\end{array}$ & 0.1945 & 0.6819 & 0.5418 \\
\cline { 2 - 6 } & Bayesian estimates & $\begin{array}{c}2.4310 \\
(0.6227)\end{array}$ & 0.001 & 1.5288 & 0.9920 \\
\hline
\end{tabular}

MLE = maximum likelihood estimation; SE = standard error; RMSE = root mean square error; LCI = length of 95\% confidence interval; $\mathrm{CP}=$ coverage probability.

Table 2: Model comparison using AIC and BIC

\begin{tabular}{|c|c|c|c|}
\hline Model & $\ln (\mathrm{L})$ & AIC & $\mathrm{BIC}$ \\
\hline Inverted exponentiated Weibull & -21.5838 & 45.4677 & 49.9701 \\
\hline Exponentiated inverted Weibull & -22.4673 & 46.9348 & 51.7371 \\
\hline Exponentiated Weibull & -47.7840 & 97.5681 & 102.3704 \\
\hline Inverse Weibull & -25.9174 & 53.8347 & 58.6371 \\
\hline Transmuted Weibull & -602.907 & 1207.8140 & 1212.6160 \\
\hline Transmuted generalized exponential & -123.3269 & 248.6538 & 253.4562 \\
\hline
\end{tabular}

and

$$
f_{\mathrm{TGE}}(x \mid \alpha, \beta, \lambda)=\alpha \beta\left(1-e^{-\beta x}\right)^{\alpha-1} e^{-\beta x}\left[1+\lambda-2 \lambda\left(1-e^{-\beta x}\right)^{\alpha}\right],
$$

where $\alpha$ is shape, $\beta$ is scale, $\lambda$ is transmuting parameter, respectively. Khan et al. $(2015,2017)$ investigated TW and TGE distributions, respectively. For the model selection, the criteria used are Akaike information criterion (AIC) and Bayesian information criterion (BIC) as:

$$
\mathrm{AIC}=2 k-2 \ln (L) \quad \text { and } \quad \mathrm{BIC}=-2 \ln (L)+k \ln (n),
$$

where $k$ is the number of parameters in the model and $L$ is the maximized value of the likelihood function for the model. The AIC is the measure of the relative quality of a statistical model for a given set of data. BIC is also a criterion for model selection among a finite set of models and is closely related to the AIC. In Table 2, the IEW model is more appropriate in terms of AIC and BIC since the values of AIC and BIC of the IEW model were smallest among the competing models. Therefore, we can conclude that the performance of the IEW model is better.

\subsection{Real data analysis}

In this section, we demonstrate the superiority of the proposed distribution $\operatorname{IEW}(\alpha, \beta, \lambda)$ using two real data sets in reliability engineering to select the best model among the competing models.

The first data arose in tests on the endurance of deep groove ball bearings which is from Lawless (1982, p. 288). They were discussed by Gupta and Kundu (1999) about Generalized exponential 


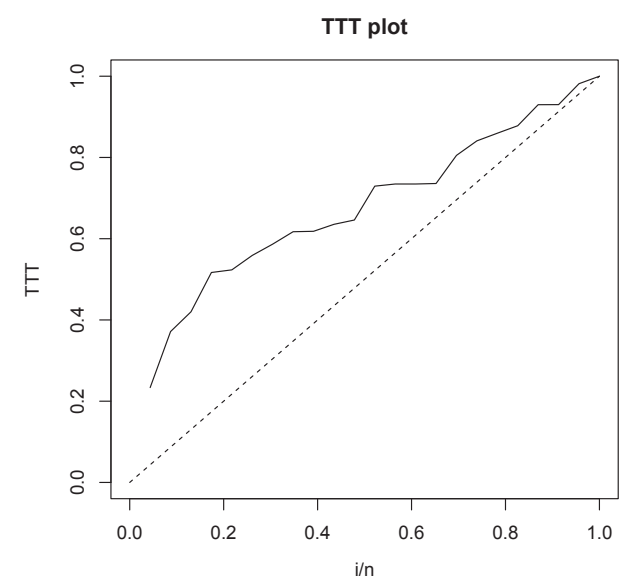

Figure 3: The TTT plot for the endurance of deep groove ball bearings data. TTT = total time on test.

Table 3: MLEs and LCIs of the model parameters for the endurance of deep groove ball bearings data, the AIC, and BIC measures

\begin{tabular}{|c|c|c|c|c|c|}
\hline Model & $\alpha(\mathrm{LCI})$ & $\beta(\mathrm{LCI})$ & $\lambda(\mathrm{LCI})$ & AIC & $\mathrm{BIC}$ \\
\hline Inverted exponentiated Weibull & $0.3350(0.070)$ & $218.2934(21.0560)$ & $28.8637(7.035)$ & 231.9890 & 235.3950 \\
\hline Exponentiated inverted Weibull & $1.8344(0.223)$ & 1.0000 & $1240.4882(99.102)$ & 237.5640 & 240.9710 \\
\hline Exponentiated Weibull & $1.3695(0.133)$ & $0.0007(4.2350)$ & $0.0015(10.235)$ & 527.6103 & 531.8813 \\
\hline Inverse Weibull & $0.3247(0.229)$ & 1.0000 & 1.0000 & 320.7850 & 323.0560 \\
\hline Transmuted Weibull & $3.5451(2.901)$ & $42127.5907(0.2340)$ & $12707.2208(30.284)$ & 760.2790 & 763.6860 \\
\hline Transmuted generalized exponential & $0.4503(0.368)$ & $287.1737(1.9345)$ & $2153.0926(10.347)$ & 310.3330 & 306.9270 \\
\hline
\end{tabular}

MLE = maximum likelihood estimation; LCI = length of 95\% confidence interval; AIC = Akaike information criterion; $\mathrm{BIC}=$ Bayesian information criterion.

distribution. The data set is:

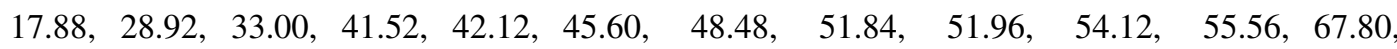
$68.64,68.64,68.88,84.12,93.12,98.64,105.12,105.84,127.92,128.04,173.40$.

Figure 3 provides a total time on test (TTT) plot for the durability of deep groove ball bearings data. Because the plot is concave and lying above the line, it means that its distribution may have an increasing hazard rate. Therefore it can be properly accommodated by a $\operatorname{IEW}(\alpha, \beta, \lambda)$ model with increasing failure rate. For comparison purposes, we consider fitting other competing distributions such as EIW, EW, IW, TW, and TGE distribution. The MLEs of the parameters, LCIs, AIC, and BIC for the fitted models are displayed in Table 3.

Table 3 says that IEW model is better than other models by comparing the values of AIC and BIC. As a result of comparing the LCI of the parameters, among the confidence intervals for $\alpha$ and $\lambda$, the LCI of the IEW model is the shortest. And among the LCI for $\beta$, the LCI of the TGE model is the shortest. We also perform likelihood ratio test (LRT) of $H_{0}: \beta=1$ (EIW model) vs $H_{1}: \beta \neq 1$ (IEW model). The LR statistic for testing the hypotheses $H_{0}$ vs. $H_{1}$ is 7.576 . LR statistic is larger than 6.634 which is critical region for significance level 0.01. So we reject the null hypotheses $H_{0}: \beta=1$ (EIW model). The plots of the fitted IEW, EIW, EW, IW, TW, and TGE densities are shown in Figure 4.

The second data set is from Birnbaum and Saunders (1969) on the fatigue life of 6061-T6 aluminum coupons cut parallel to the direction of rolling and oscillated at 18 cycles per second. The data 


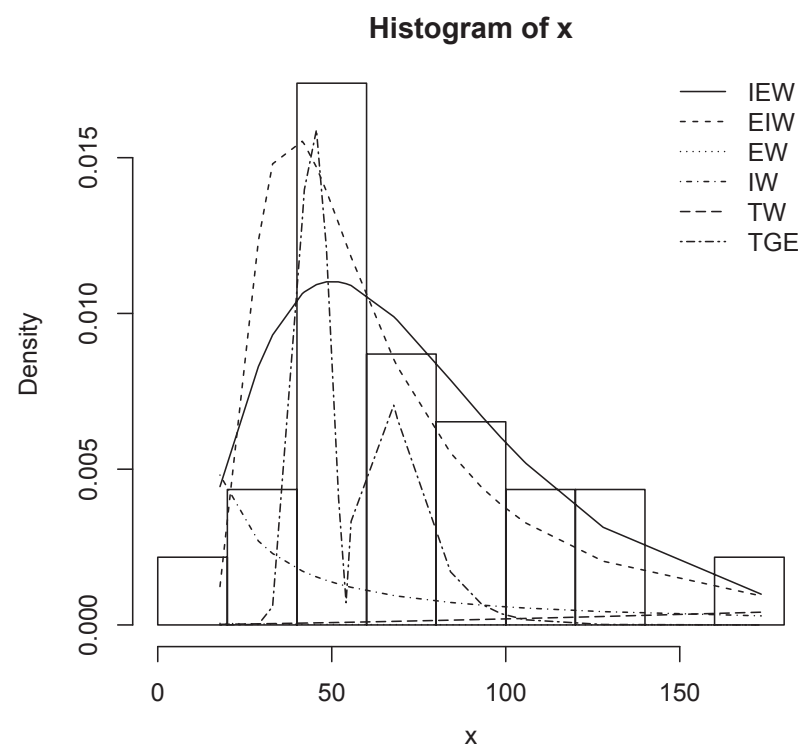

Figure 4: Fitted IEW, EIW, EW, IW, TW, and TGE densities for the endurance of deep groove ball bearings data. $I E W=$ inverted exponentiated Weibull; EIW = exponentiated inverted Weibull; EW = exponentiated Weibull; $I W=$ inverse Weibull; TW = transmuted Weibull; TGE = transmuted generalized exponential.

set is:

70, 90, 96, 97, 99, 100, 103, 104, 104, 105, 107, 108, 108, 108, 109, 109,

$112,112,113,114,114,114,116,119,120,120,120,121,121,123,124,124$,

$124,124,124,128,128,129,129,130,130,130,131,131,131,131,131,132$,

132, 132, 133, 134, 134, 134, 134, 136, 136, 137, 138, 138, 138, 139, 139, 141,

$141,142,142,142,142,142,142,144,144,145,146,148,148,149,151,151$,

$152,155,156,157,157,157,157,158,159,162,163,163,164,166,166,168$,

$170,174,201,212$.

Figure 5 provides a TTT plot for the fatigue life of 6061-T6 data. As similar to Figure 3, Figure 5 also shows that its plot is concave and lying above the line; therefore, and so, its distribution may have an increasing hazard rate. It can also be properly accommodated by a IEW model with an increasing failure rate.

Table 4 displays the MLEs and its LCIs of the parameters as well as the values of AIC and BIC for the fitted models. Table 4 says that IEW model is better than other models by comparing AIC and BIC. As a result of comparing the LCIs of the parameters, among the LCIs for $\alpha$, the LCI of the IEW model is the shortest; in addition, the LCIs for $\beta$ and $\lambda$, the LCI of the EW model is the shortest. We perform LRT of $H_{0}: \beta=1$ (EIW model) vs. $H_{1}: \beta \neq 1$ (IEW model). The LR statistic for testing the hypotheses $H_{0}$ vs. $H_{1}$ is 27.509 . LR statistic is larger than 6.634 which is a critical region for significance level 0.01. So we reject the null hypotheses $H_{0}: \beta=1$ (EIW model). The plots of the fitted IEW, EIW, EW, IW, TW, and TGE densities are shown in Figure 6. 


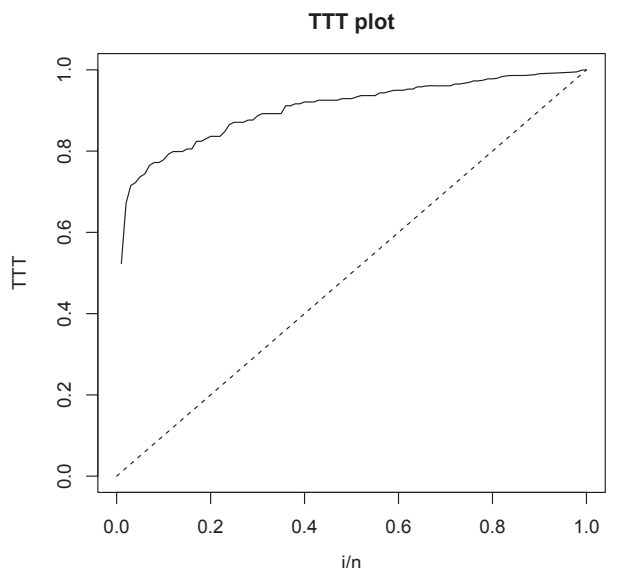

Figure 5: The TTT plot for the 6061-T6 data. TTT = total time on test.

Table 4: MLEs of the model parameters for 6061-T6 data, the AIC and BIC measures

\begin{tabular}{|c|c|c|c|c|c|}
\hline Model & $\alpha(\mathrm{LCI})$ & $\beta$ (LCI) & $\lambda(\mathrm{LCI})$ & AIC & BIC \\
\hline Inverted exponentiated Weibull & $0.90930(0.035)$ & $262.5843(10.3541)$ & $501.0968(17.427)$ & 920.356 & 928.172 \\
\hline Exponentiated inverted Weibull & $5.04270(0.074)$ & 1.0000 & $3146.4000(32.120)$ & 947.865 & 955.681 \\
\hline Exponentiated Weibull & $1.06400(0.037)$ & $50.2247(10.006)$ & $230.2991(23.015)$ & 237.564 & 1240.971 \\
\hline Inverse Weibull & $0.27650(0.082)$ & 1.0000 & 1.0000 & 1559.361 & 1564.572 \\
\hline Transmuted Weibull & $3.31890(0.731)$ & $53.8542(1.274)$ & $2094.2543(27.538)$ & $\infty$ & $\infty$ \\
\hline Transmuted generalized exponential & $0.59138(0.374)$ & $576.9850(1.637)$ & $5120.2012(22.052)$ & 1324.593 & 1317.336 \\
\hline
\end{tabular}

MLE = maximum likelihood estimation; LCI = length of 95\% confidence interval; AIC = Akaike information criterion; $\mathrm{BIC}=$ Bayesian information criterion.

\section{Conclusion}

We propose a new model which is IEW distribution that extends EW distribution introduced by Mudholkar et al. (1993). Some properties are introduced and plots of pdf and hazard functions are displayed to show the flexibility of IEW distribution. We propose MLEs using the Newton Raphson method and Bayesian estimation using Gibbs sampler. We present the AIC and BIC to compare the IEW modes and special sub-models. We also present the use of LR statistics to compare the fit of the IEW with EIW which is a special sub-model. Finally, we fit the IEW model to two real data sets to show the flexibility and potential of the IEW distribution.

\section{Appendix: Proof of Theorem}

Proof of Theorem 1: Let $g_{1}(\alpha ; \beta, \lambda)$ be the RHS of (4.8). For $\beta=1$, let $w_{1}=\sum_{i=1}^{n} \lambda x_{i}^{-\alpha} \log x_{i}$. Then $\lim _{\alpha \rightarrow 0} w_{1}=\sum_{i=1}^{n} \lambda \log x_{i}$ and $\lim _{\alpha \rightarrow \infty} w_{1}=0$, where $x_{i}>1$. Therefore,

$$
g_{1}(\alpha ; \beta, \lambda)=\frac{n}{\alpha}-\sum_{i=1}^{n} \log x_{i}+w_{1}>\frac{n}{\alpha}-\sum_{i=1}^{n} \log x_{i}+\lim _{\alpha \rightarrow \infty} w_{1}
$$

thus, $g_{1}(\alpha ; \beta, \lambda)>0$ if $\alpha>n /\left(\sum_{i=1}^{n} \log x_{i}\right)$. 


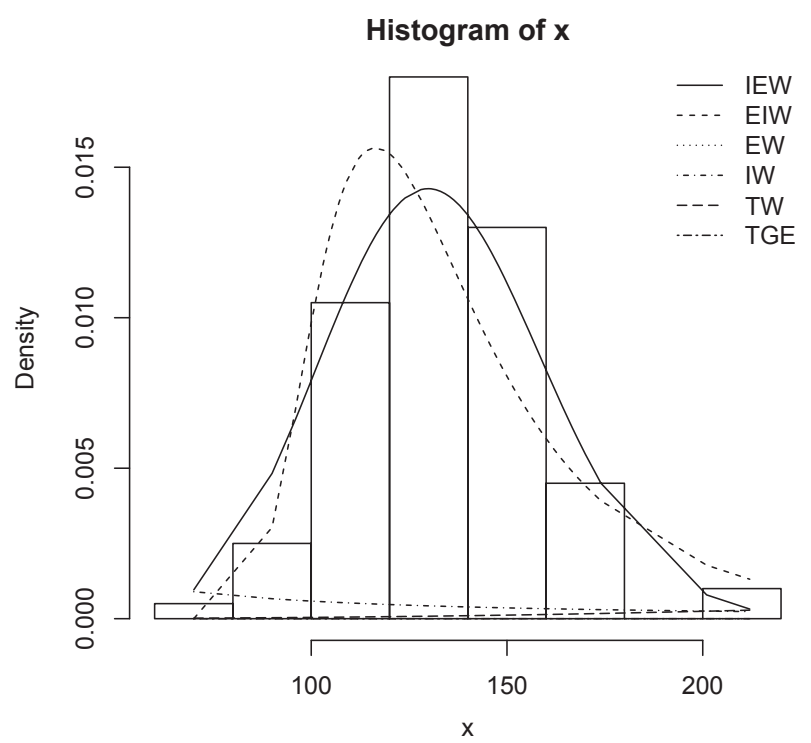

Figure 6: Fitted IEW, EIW, EW, IW, TW, and TGE densities for the 6061-T6 data. IEW = inverted exponentiated Weibull; EIW = exponentiated inverted Weibull; $E W=$ exponentiated Weibull; $I W=$ inverse Weibull; $T W=$ transmuted Weibull; TGE = transmuted generalized exponential.

On the other side

$$
g_{1}(\alpha ; \beta, \lambda)=\frac{n}{\alpha}-\sum_{i=1}^{n} \log x_{i}+w_{1}<\frac{n}{\alpha}-\sum_{i=1}^{n} \log x_{i}+\lim _{\alpha \rightarrow 0} w_{1},
$$

hence, $g_{1}(\alpha ; \beta, \lambda)<0$ if $\alpha<n /\left((1-\lambda) \sum_{i=1}^{n} \log x_{i}\right)$. The roots of $g_{1}(\alpha ; \beta, \lambda)=0$ lie in the interval $\left[n /\left((1-\lambda) \sum_{i=1}^{n} \log x_{i}\right), n /\left(\sum_{i=1}^{n} \log x_{i}\right)\right]$.

For $\beta \neq 1, \lim _{\alpha \rightarrow 0} g_{1}=\infty$ and $\lim _{\alpha \rightarrow \infty} g_{1}=-\sum_{i=1}^{n} \log x_{i}<0$, where $x_{i}>1$, thus, $g_{1}(\alpha ; \beta, \lambda)=0$ has at least one root.

Proof of Theorem 2: Let $g_{2}(\beta ; \alpha, \lambda)$ be the RHS of (4.9). Consider that $\lim _{\beta \rightarrow 0} g_{2}=\infty$ and $\lim _{\beta \rightarrow \infty} g_{2}$ $=\sum_{i=1}^{n} \log \left(1-e^{-\lambda x^{-\alpha}}\right)<0$. To prove the uniqueness we show that $\left(\partial g_{2}(\beta ; \alpha, \lambda)\right) / \partial \beta=-n / \beta^{2}<0$. Since the second derivative is negative, the solution to the first order condition determines the unique maximizer of the likelihood function, and is the maximum likelihood estimator.

Proof of Theorem 3: Let $g_{3}(\beta ; \alpha, \lambda)$ be the RHS of (5.1). $\lim _{\lambda \rightarrow 0} g_{3}(\beta ; \alpha, \lambda)=\infty$ and $\lim _{\beta \rightarrow \infty} g_{3}$ $(\beta ; \alpha, \lambda)=-\sum_{i=1}^{n} x_{i}<0$. Therefore $g_{3}(\beta ; \alpha, \lambda)=0$ has at least one root.

\section{References}

Birnbaum ZW and Saunders SC (1969). Estimation for a family of life distributions with applications to fatigue, Journal of Applied Probability, 6, 328-347.

Chib S and Greenberg E (1995). Understanding the Metropolis-Hastings algorithm, The American Statistician, 49, 327-335.

De Gusmão FRS, Ortega EMM, and Cordeiro GM (2011). The generalized inverse Weibull distribution, Statistical Papers, 52, 591-619. 
De Gusmão FRS, Ortega EMM, and Cordeiro GM (2012). Reply to the "Letter to the Editor" of M. C. Jones, Statistical Papers, 53, 253-254.

Elbatal I, Condino F, and Domma F (2016). Reflected generalized beta inverse Weibull distribution: definition and properties, Sankhya B, 78, 316-340.

Flaih A, Elsalloukh H, Mendi E, and Milanova M (2012). The exponentiated inverted Weibull distribution, Applied Mathematics and Information Sciences, 6, 167-171.

Gelfand AE and Smith AFM (1990). Sampling-based approaches to calculating marginal densities, Journal of the American Statistical Association, 85, 398-409.

Gupta RD and Kundu D (1999). Theory \& methods: generalized exponential distributions, Australian and New Zealand Journal of Statistics, 41, 173-188.

Jones MC (2012). Letter to the Editor, Statistical Papers, 53, 251.

Khan MS, King R, and Hudson IL (2015). Transmuted generalized exponential distribution: A generalisation of the exponential distribution with applications to survival data, Communications in Statistics - Simulation and Computation, Manuscript just-accepted for publication.

Khan MS, King R, and Hudson IL (2017). Transmuted Weibull distribution: properties and estimation, Communications in Statistics - Theory and Methods, 46, 5394-5418.

Krishna H and Kumar K (2013). Reliability estimation in generalized inverted exponential distribution with progressively type II censored sample, Journal of Statistical Computation and Simulation, 83, 1007-1019.

Lawless JF (1982). Statistical Models and Methods for Lifetime Data, Wiley, New York.

Lee S (2014). Bayesian Estimation of Parameters of Inverted Exponentiated Weibull Distribution (Master's Thesis), Pusan National University, Pusan.

Marshall AW and Olkin I (2007). Lifetime Distributions: Structure of Non-parametric, Semiparametric, and Parametric Families, Springer, New York.

Mudholkar GS and Srivastava DK (1993). Exponentiated Weibull family for analyzing bathtub failure-rate data, IEEE Transactions on Reliability, 42, 299-302.

Rastogi MK and Tripathi YM (2014). Estimation for an inverted exponentiated Rayleigh distribution under type II progressive censoring, Journal of Applied statistics, 41, 2375-2405.

Singh SK, Singh U, and Kumar D (2013). Bayesian estimation of parameters of inverse Weibull distribution, Journal of Applied statistics, 40, 1597-1607.

Singh U, Gupta PK, and Upadhyay SK (2002). Estimation of exponentiated Weibull shape parameters under LINEX loss function, Communications in Statistics - Simulation Computation, 31, 523537.

Singh U, Gupta PK, and Upadhyay SK (2005). Estimation of three-parameter exponentiated-Weibull distribution under type-II censoring, Journal of Statistical Planning and Inference, 134, 350372. 